\title{
Finite Element Analysis of Active Vibration Isolation Using Vibrational Power as a Cost Function
}

\author{
Carl Q. Howard and Colin H. Hansen \\ Department of Mechanical Engineering, The University of Adelaide, South Australia 5005, Australia
}

\begin{abstract}
An active vibration isolation system comprised of a simply-supported beam and a rigid mass mounted on an active isolator is analyzed using Finite Element Analysis. The cost function which is minimized is the vibrational power transmitted from the vibrating mass into the beam. The analysis shows that moments can generate negative power transmission values along a translational axis. It is shown that a control strategy which minimizes the power transmission along a translational axis and neglects the power transmission due to moments can produce higher beam vibration levels than without control. It is shown by example that the minimization of squared acceleration or squared force in the vertical direction at the base of the isolator, performs nearly as well as the minimization of total power transmission (along translational and rotational axes). It is shown that the cost function of translational power transmission along the vertical axis can have negative values, when rotational moments are present. In these situations, the cost function of squared power transmission along the vertical axis will have a locus of filter weights where the squared power transmission is zero along the vertical axis. The optimum set of filter weights corresponding to the minimization of squared acceleration or squared force along a vertical axis, is a point which lies on this locus. It is shown that a point exists on this locus, where the control effort is also minimized. At this point, the control effort is less than that required when the squared acceleration or squared force along the vertical axis is minimized. However, at the point where squared power transmission along the vertical axis and the control effort is minimized, the total power transmission is not necessarily minimized and generally not as small as achieved by just minimizing squared force or squared acceleration in the vertical $Z$ direction at the base of the isolator. Two adaptive control algorithms are suggested for finding the optimum filter weights which minimize the squared power transmission and the control effort. The first algorithm uses Newton's method to minimize the control effort by moving the filter weights along a constant power level on the error surface without causing an increase in the residual error. A second method is suggested which alternates the filter weight updates between a partial leaky filtered- $x$ LMS algorithm and the standard filtered- $x$ LMS algorithm. This results in a zigzag path of the filter weights and a slightly
\end{abstract}

\section{INTRODUCTION}

In a typical vibration isolation system, a vibrating rigid body provides a power source which is dissipated by an isolator and a support structure. Vibrational power is transferred by translational forces and rotational moments. It has been shown in the literature that in the calculation of vibrational power transmission (or structural intensity) at the intersection of an active isolator and support structure, the inclusion of power from rotational moments can act to cancel the contribution of power from translational forces. ${ }^{1,2}$ Power transmission from moments is converted into translational power transmission upon reflection at the supports, and interacts with the translational power transmission due to translational forces. This can result in negative values of vibratory power transmission (that is, power reversal) along a translational axis.

Previous experimental work reported by the authors ${ }^{3}$ showed that for a vibrating mass actively isolated from a simply-supported beam, there were frequencies for which the vertical power transmission under active control was worse than for the passive case. An accelerometer and force transducer combination was used to measure the power transmission from the isolator into the beam. A heterodyning technique was used to combine velocity and force signals at the base of the isolator, into a signal which was proportional to the vertical vibrational power transmission at the driving frequency. ${ }^{3}$ It was reported that power transmission from moments was suspected to be the cause of the measurement of negative power transmission.

In experimental work on active vibration isolation, the measurement of moments is often omitted because their contribution is considered negligible or because of the unavailability of suitable transducers. This paper demonstrates through Finite Element Analysis that a control strategy which minimizes the power transmission into a support structure, when the power transmission due to small moments is neglected, can result in total vibrational power levels greater than without control.

Gardonio $^{\mathbf{4 , 5}}$ has analyzed the power transmission of a vibrating rigid mass isolated from a plate using two active mounts. Gardonio showed that minimization of the out of plane component of power, when power transmission due to moments was omitted, caused a "power circulation" phenomenon, where power was drawn into the plate and then re-absorbed by the active mounts. Power circulation caused greater vibration levels in the plate than without active control. Gardonio's work used two different types of cost functions. The first was the out of plane power transmission, which was capable of negative values and the second was the weighted sum of the out of plane squared acceleration and squared force, which is positive definite. The weighting factor was applied to the squared force error signal so that it was the same order of magnitude as the squared velocity signal. In this case, the weighting factor was chosen to be the square of the point mobility of the receiving structure. 